\title{
Combining two radiopeptide therapies improves survival
}

Neuroendocrine tumours frequently express the somatostatin receptor, a therapeutic target for radiopeptide therapy. The somatostatin analogue DOTA-TOC was developed and introduced into the clinic at the University Hospital Basel in 1997. Martin Walter has searched to optimize ways to deliver $\beta$-emitting radioisotopes in patients with metastatic neuroendocrine tumours because the radionuclides ${ }^{90}$ Yttrium $\left({ }^{90} \mathrm{Y}\right)$ and ${ }^{177}$ Lutetium $\left({ }^{177} \mathrm{Lu}\right)$ have shown

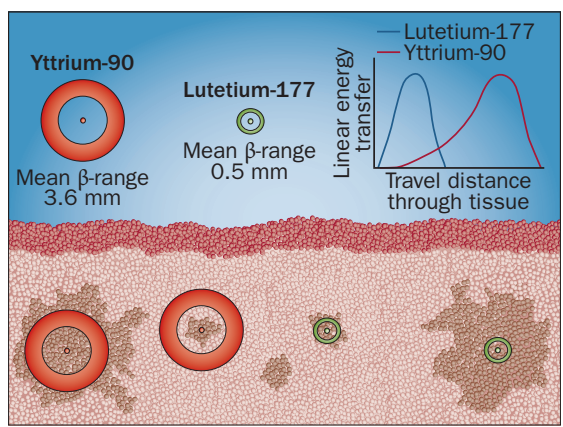

Modified from image supplied by M. Walter synergistic effects in animal models. Walter's team have now shown that combining the radionucleotide-labelled analogues $\left[{ }^{90} \mathrm{Y}\right.$-DOTA]-TOC and [ ${ }^{177} \mathrm{Lu}$-DOTA]-TOC improved overall survival compared with $\left[{ }^{90} \mathrm{Y}-\mathrm{DOTA}\right]-\mathrm{TOC}$ in patients with neuroendocrine tumours.

The different path ranges of the two radionuclides result in complementary characteristics; ${ }^{90} \mathrm{Y}$ transfers most of its energy to macrometastatic lesions, whereas ${ }^{177} \mathrm{Lu}$ transfers most of its energy to micrometastases. The researchers hypothesized that the combination of these radionuclides would be more efficacious than either of them alone because both large and small lesions could be targeted.

A total of 486 patients from 80 centres in 19 countries completed three or more treatment cycles. Walter highlights the main findings of the study, "patients receiving $\left[{ }^{90} \mathrm{Y}-\mathrm{DOTA}\right]-\mathrm{TOC}$ plus $\left[{ }^{177} \mathrm{Lu}-\mathrm{DOTA}\right]-\mathrm{TOC}$ had a significantly longer survival than those receiving [90Y-DOTA]-TOC alone (5.51 years versus 3.96 years)." Renal toxicity is a known adverse effect associated with this treatment, but as Walter notes, "the rates of severe haematological toxicities and severe renal toxicity were comparable in both groups".

He continues, "to our knowledge, this study is the first to show beneficial effects of using two isotopes for DOTA-TOC therapy". As this was a cohort study and unknown prognostic factors might be unbalanced between the two treatment groups, a randomized study is now needed to confirm the results of this promising therapy combination.

\section{Lisa Hutchinson}

Original article Villard, L. et al. Cohort study of somatostatin-based radiopepdide therapy with [ ${ }^{90} \mathrm{Y}$-DOTA] TOC versus [ ${ }^{90} \mathrm{Y}$-DOTA]-TOC plus [ $\left.{ }^{177} \mathrm{Lu}-\mathrm{DOTA}\right]-\mathrm{TOC}$ in neuroendocrine cancers. J. Clin. Oncol. doi:10.1200/ JC0.2011.37.2151 (2012) 